OPEN ACCESS

Edited by: Alfredo Conti,

University of Bologna, Italy

Reviewed by:

Antonio Pontoriero,

University of Messina, Italy

Scott Soltys,

Stanford University, United States

*Correspondence:

Tanja Eichkorn

tanja.eichkorn@med.uni-heidelberg.de

Specialty section:

This article was submitted to Neuro-Oncology and

Neurosurgical Oncology,

a section of the journal

Frontiers in Oncology

Received: 08 September 2021 Accepted: 21 October 2021

Published: 17 November 2021

Citation:

Eichkorn T, Regnery S, Held T, Kronsteiner D, Hörner-Rieber J,

El Shafie RA, Herfarth K, Debus J and

König $L$ (2021) Effectiveness and Toxicity of Fractionated Proton Beam

Radiotherapy for Cranial Nerve

Schwannoma Unsuitable for

Stereotactic Radiosurgery.

Front. Oncol. 11:772831.

doi: 10.3389/fonc.2021.772831

\section{Effectiveness and Toxicity of Fractionated Proton Beam Radiotherapy for Cranial Nerve Schwannoma Unsuitable for Stereotactic Radiosurgery}

\author{
Tanja Eichkorn ${ }^{1,2^{*}}$, Sebastian Regnery ${ }^{1,2}$, Thomas Held ${ }^{1,2}$, Dorothea Kronsteiner ${ }^{3}$, \\ Juliane Hörner-Rieber ${ }^{1,2}$, Rami A. El Shafie ${ }^{1,2}$, Klaus Herfarth ${ }^{1,2}$, \\ Jürgen Debus ${ }^{1,2,4,5,6}$ and Laila König ${ }^{1,2}$
}

\begin{abstract}
${ }^{1}$ Department of Radiation Oncology, Heidelberg University Hospital, Heidelberg, Germany, ${ }^{2}$ National Center for Radiation Oncology (NCRO), Heidelberg Institute for Radiation Oncology (HIRO), Heidelberg, Germany, ${ }^{3}$ Institute of Medical Biometry and Informatics, Heidelberg University, Heidelberg, Germany, ${ }^{4}$ Clinical Cooperation Unit Radiation Oncology (E050), German Cancer Research Center (dkfz), Heidelberg, Germany, ${ }^{5}$ National Center for Tumor diseases (NCT), Heidelberg, Germany,

${ }^{6}$ Deutsches Konsortium für Translationale Krebsforschung (DKTK), Partner Site Heidelberg, German Cancer Research Center (dkfz), Heidelberg, Germany
\end{abstract}

Purpose: In this benign tumor entity, preservation of cranial nerve function is of special importance. Due to its advantageous physical properties, proton beam radiotherapy (PRT) is a promising approach that spares healthy tissue. Could PRT go along with satisfactory preservation rates for cranial nerve function without compromising tumor control in patients with cranial nerve schwannoma unsuitable for stereotactic radiosurgery?

Methods: We analyzed 45 patients with cranial nerve schwannomas who underwent PRT between 2012 and 2020 at our institution. Response assessment was performed by $\mathrm{MRI}$ according to RECIST 1.1, and toxicity was graded following CTCAE 5.0.

Results: The most common schwannoma origin was the vestibulocochlear nerve with $82.2 \%$, followed by the trigeminal nerve with $8.9 \%$ and the glossopharyngeal nerve as well as the vagal nerve, both with each $4.4 \%$. At radiotherapy start, $58 \%$ of cranial nerve schwannomas were progressive and $95.6 \%$ were symptomatic. Patients were treated with a median total dose of 54 Gy RBE in 1.8 Gy RBE per fraction. MRI during the median follow-up period of 42 months (IQR 26-61) revealed stable disease in 93.3\% of the patients and partial regression in $6.7 \%$. There was no case of progressive disease. New or worsening cranial nerve dysfunction was found in $20.0 \%$ of all patients, but always graded as CTCAE ㅇ-II. In seven cases (16\%), radiation-induced contrast enhancements (RICE) were detected after a median time of 14 months (range 2-26 months). RICE were asymptomatic $(71 \%)$ or transient symptomatic (CTCAE II; 29\%). No CTCAE III/IV toxicities were observed. Lesions regressed during the follow-up period in three of the seven cases, and no lesion progressed during the follow-up period. 
Conclusion: These data demonstrate excellent effectiveness with $100 \%$ local control in a median follow-up period of 3.6 years with a promising cranial nerve functional protection rate of $80 \%$. RICE occurred in $16 \%$ of the patients after PRT and were not or only mildly symptomatic.

Keywords: acoustic neuroma, vestibular schwannoma, radiation-induced contrast enhancements (RICE), pseudoprogression, radiation necrosis

\section{INTRODUCTION}

Schwannomas are usually benign and slowly growing nerve sheet tumors that arise from the Schwann cells lining of peripheral nerves. They are most commonly located in the intradural extramedullary space and therefore affect mainly cranial or spinal nerves (1). Vestibular schwannomas (also known as acoustic neuromas) that commonly arise from the vestibular portion of the eighth cranial nerve account for most schwannomas. The overall incidence is approximately 1 in 100,000 persons per year in Western countries (2). While sporadic schwannomas are rare, they occur frequently in patients with neurofibromatosis. Bilateral vestibular schwannomas in children with neurofibromatosis put them at risk for complete deafness (3-6).

In the past decades, the most effective treatment for progressive cranial nerve schwannomas has been complete surgical resection. While local control rates were excellent, injuries of the affected or adjacent cranial nerves make the treatment of cranial nerve schwannomas a major challenge. A large surgical trial reports in vestibular schwannoma a functional hearing deterioration in up to $60.5 \%$ after surgical resection. So, cranial nerve injuries cause a relatively high morbidity compared to the excellent oncologic prognosis $(7,8)$. Due to the slow progression of schwannomas, the "watch and wait strategy" can be a legitimate treatment option for selected patients (9). Therapy sequelae like hearing impairment and tinnitus, vertigo and gait disturbances, facial nerve palsy, facial pain or hypesthesia, swallowing difficulties, or others also impair the quality of life. Therefore, treatment decision needs to be made carefully, and research on nerve-saving techniques is necessary.

A promising new technique was developed with stereotactic radiosurgery. Even if large prospective data are still lacking, stereotactic radiosurgery (SRS) seems to be superior regarding the risk of cranial nerve damage in schwannomas smaller than $3 \mathrm{~cm}$ with excellent local control rates compared to surgical resection and is therefore taken into consideration in the European Association of Neuro-Oncology (EANO) guidelines (9).

But concerns about the induction of malignant transformation of schwannomas or secondary malignancy induction after stereotactic radiosurgery still exist, even if there is no evidence for these concerns in literature, neither for stereotactic radiosurgery nor for particle beam radiotherapy (10-13). Proton beam radiotherapy (PRT) is a promising approach to better spare healthy surrounding tissue and therefore might contribute to the reduction of side effects in patients unsuitable for SRS due to the advanced tumor stage.

The major concern with proton beam radiotherapy is the risk of radiation-induced contrast enhancements (RICE), as known from, e.g., glioma trials $(14,15)$. RICE are defined by new brain lesions outside the tumor volume related to cerebral irradiation that are usually contrast-enhancing and not caused by the tumor. These RICE are usually transient blood-brain barrier disruptions and rarely real necrosis.

To date, there are only scarce data on PRT for schwannomas. One study investigated efficacy and toxicity rates in 94 patients who underwent fractionated PRT for vestibular schwannoma. These data demonstrated excellent local control rates and a dosedependent risk for hearing deterioration of $36 \%$ to $56 \%$ with doses from 50.4 to $54 \mathrm{~Gy}$, while the risk for damage of other cranial nerves was $5 \%$ (16). A case series supported the fact that fractionated PRT for vestibular schwannoma is well tolerated and provides good local control (17). A retrospective cohort study investigated proton (!) beam stereotactic radiosurgery and reported a 5-year tumor control rate of $95 \%$ and a dose dependency for facial neuropathy (18). No data at all exist on PRT for other schwannomas except for vestibular schwannomas.

This study investigates the effectiveness and toxicity of fractionated proton beam radiotherapy for cranial nerve schwannomas that were unsuitable for SRS. Is excellent tumor control achievable without major sequelae?

\section{PATIENTS AND METHODS}

\section{Patient Characteristics}

According to its physical properties, in patients with large target volumes (for skull base schwannoma defined by T3-T4 tumors) or tumors in close proximity to the brain stem or other cranial nerves or if patients could not undergo surgery, PRT was chosen, as it is suspected that, in these cases, PRT is more suitable than stereotactic radiosurgery (SRS) and might be more suitable than fractionated photon radiotherapy. Potential physical superiority of protons over photons is well investigated in literature, but potential clinical superiority of fractionated proton radiotherapy over fractionated photon radiotherapy for cranial nerve schwannomas has never been proven. This study shall provide some clinical data on fractionated proton radiotherapy for cranial nerve schwannomas. Figure 1 demonstrates how decision was made to treat patients with fractionated PRT. We finally included 45 patients with cranial nerve schwannomas who underwent fractionated PRT between 2012 and 2020 at our ion beam therapy center. Patient and treatment data were extracted from a clinical database maintained at our institution and from medical and official records. The first follow-up cMRI was performed 2-3 months after finishing radiotherapy. If no 


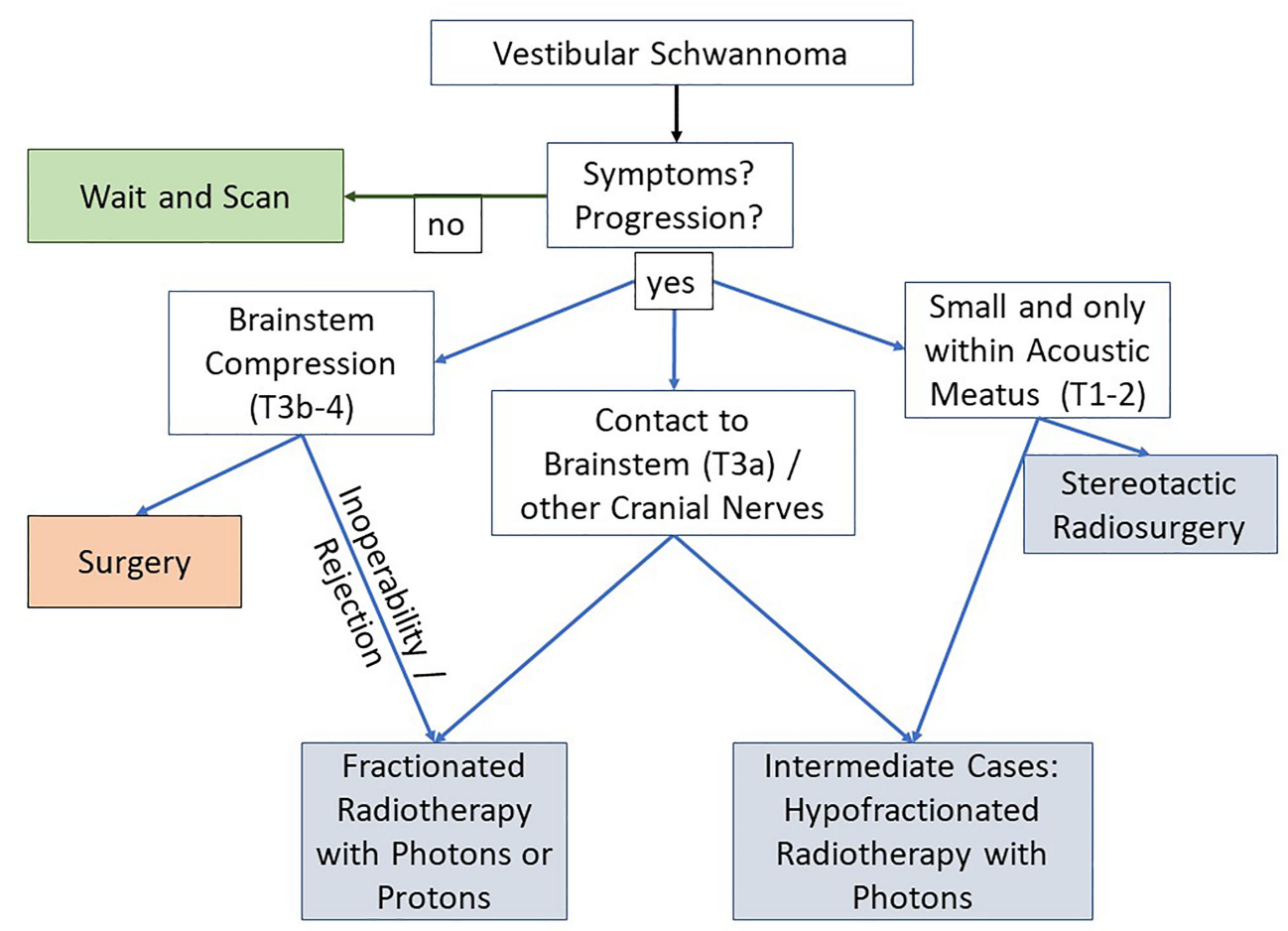

FIGURE 1 | Treatment decision-making in our study cohort exemplarily for vestibular schwannoma. For other cranial nerve neuromas than vestibular schwannoma, similar decision algorithms were used.

abnormalities were found, the following cMRIs were done in time intervals of 6-12 months thereafter. Exploration of RICE risk factors included all available treatment and patient characteristics as listed in the tables.

\section{Planning and Treatment Features}

Immobilization was ensured by using individually shaped thermoplastic masks in the head first-supine position. In this positioning, a computed tomography (CT) scan with 3-mm slice thickness as well as a cranial magnetic resonance tomography (cMRI) with contrast were acquired for treatment planning. Gross tumor volume (GTV) comprised the contrast enhanced schwannoma in T1-weightened cMRI. A planning target volume (PTV) margin of $3 \mathrm{~mm}$ isotopically was added to account for geometrical uncertainties and physical beam inaccuracies. Treatment planning followed the principle of irradiation dose being as low as reasonably achievable (ALARA) without compromising PTV coverage. Dose prescription to the target volume was performed according to the constraints of ICRU report 50 and 62. Normal tissue constraints according to QUANTEC and Emami et al. $(19,20)$ were adhered to and sometimes adapted according to the preserved cranial nerve function, e.g., hearing function. Active beam application using raster-scanning technique with a spot size between 8 and $30 \mathrm{~mm}$ full width at half maximum (FWHM), with 2-3 mm of overlap in lateral $(\mathrm{dx}, \mathrm{dy})$ and longitudinal $(\mathrm{dz})$ directions and synchrotron energy (48-250 MeV), was used, the active change of energy being available in 256 discrete steps, using two to three treatment beams under daily image guidance, orthogonal $\mathrm{x}$-rays mounted on a ceiling robotic arm and with $2 \mathrm{D}-3 \mathrm{D}$ image registration for the robotic couch position correction. Either single-beam optimization (SBO) or multibeam optimization (IMPT) was used. IMPT was aimed for avoiding high-dose gradients per field, e.g., in difficult shaped targets. The final proton dose was scaled with a constant RBE factor of 1.1. Treatment was performed with five to six fractions per week. We took anatomic factors that might lead to dose uncertainties into account and used multiple beams, if needed. Furthermore, in our clinical routine, it is mandatory to regularly perform position verification scans during the treatment period with plan recalculations. So, in case of anatomical changes, e.g., in the petrous bone cavities, we adapted treatment plans. The conformity index (CI) for PTV was calculated according to the RTOG guidelines (21) by division of the CTV covered by the $95 \%$ isodose (reference isodose) and the target volume itself. A value close to 1 corresponds to ideal conformity.

\section{Endpoints}

Trial endpoints were effectiveness and toxicity of proton beam radiotherapy. Effectiveness was evaluated by "Response Evaluation Criteria in Solid Tumors" (RECIST) version 1.1 and divided into complete or partial response, stable disease, and progressive disease $(22,23)$. Schwannoma progression was defined as an increase in volume according to RECIST in the 
follow-up period without spontaneous regression in the following cranial magnetic resonance imaging (cMRI) to distinguish it from post-treatment edema. Toxicity was graded following the National Cancers Institute's Common Terminology Criteria for Adverse Events (CTCAE) (version 5.0). Cranial nerve functional impairment was assessed by history taking and physical exam. RICE was defined by a new post-treatment contrast enhancement in cMRI outside the GTV during the follow-up period. To face the risk of misinterpretation of RICE as tumor progression or the other way around, all images were reviewed independently by one radiologist and two radiation oncologists. Other toxic effects were assessed based on both medical records and imaging reports.

\section{Statistical Analysis}

Descriptive statistics for baseline variables (Tables 1, 2) and for objectives (Tables 3-5) include means (SD) and/or median (IQR and range, as appropriate) for continuous variables and absolute and relative frequencies for categorical variables. To identify influencing factors on clinical symptom improvement or deterioration in the follow-up period, a logistic regression

TABLE 1 | Patient baseline characteristics.

\begin{tabular}{|c|c|c|}
\hline & \multicolumn{2}{|c|}{$n=45[\%]$} \\
\hline \multicolumn{3}{|l|}{ Gender } \\
\hline Female & 23 & [51.1\%] \\
\hline Male & 22 & [48.9\%] \\
\hline \multicolumn{3}{|l|}{ Age at initial diagnosis (years) } \\
\hline Mean & 51 & \\
\hline Median & 51 & \\
\hline Standard deviation & 19 & \\
\hline Quartile 1-quartile 3 & $39-66$ & \\
\hline Minimum-maximum & 10-82 & \\
\hline \multicolumn{3}{|l|}{ Age at radiotherapy (years) } \\
\hline Median & 55 & \\
\hline Minimum-maximum & $18-88$ & \\
\hline \multicolumn{3}{|l|}{ Schwannoma risk factors } \\
\hline Neurofibromatosis type 2 & 5 & [11.1\%] \\
\hline \multicolumn{3}{|l|}{ Cranial nerve } \\
\hline Trigeminal nerve & 4 & [8.9\%] \\
\hline Vestibulocochlear nerve & 37 & [82.2] \\
\hline Glossopharyngeal nerve & 2 & [4.4\%] \\
\hline Vagal nerve & 2 & [4.4\%] \\
\hline \multicolumn{3}{|l|}{ Diagnostic methods } \\
\hline MRI only & 28 & [62.2\%] \\
\hline DOTATOC-PET-CT & 2 & [4.4\%] \\
\hline Partial resection/biopsy & 14 & [31.1\%] \\
\hline Complete resection & 3 & {$[6.7 \%]$} \\
\hline Radiotherapy due to recurrence & 3 & [100\%] \\
\hline \multicolumn{3}{|c|}{ Proof of progressive schwannoma before radiotherapy } \\
\hline Yes & 26 & [57.8\%] \\
\hline No & 19 & [42.2\%] \\
\hline \multicolumn{3}{|c|}{ Symptomatic schwannoma (at radiotherapy start) } \\
\hline Yes & 43 & [95.6\%] \\
\hline No & 2 & [4.4\%] \\
\hline \multicolumn{3}{|c|}{ Tumor size at radiotherapy start $(\mathrm{ml})$} \\
\hline Mean & 8 & \\
\hline Median & 5 & \\
\hline Standard deviation & 10 & \\
\hline Quartile 1-quartile 3 & $3-8$ & \\
\hline Minimum-maximum & $0.3-60$ & \\
\hline
\end{tabular}

model was applied using multiple patient and treatment characteristics: age $>55$ years, tumor volume $\geq 5 \mathrm{ml}$, technique (IMPT vs. SBO), number of beams, and fractions per week. Since this is a retrospective exploratory data analysis, $\mathrm{p}$-values are of descriptive nature. Statistical analyses are performed with the software R Version 4.0.3.

\section{RESULTS}

\section{Patient and Treatment Characteristics}

Median patient age at the beginning of radiotherapy (RT) was 55 years (range: 18-88). Gender was equally distributed. The most common schwannoma origin was the vestibulocochlear nerve with $82.2 \%$, followed by the trigeminal nerve with $8.9 \%$ and the

TABLE 2 | Treatment characteristics.

\begin{tabular}{|c|c|c|}
\hline A) Overview & \multicolumn{2}{|c|}{$n=45[\%]$} \\
\hline \multicolumn{3}{|c|}{ Primary diagnosis until radiation therapy start (months) } \\
\hline Median & 18 & \\
\hline Minimum-maximum & $2-159$ & \\
\hline \multicolumn{3}{|l|}{ Total dose (Gy RBE) } \\
\hline Mean & 54 & \\
\hline Median & 54 & \\
\hline Standard deviation & 3 & \\
\hline Quartile 1-quartile 3 & $54-56$ & \\
\hline Minimum-maximum & $40-58$ & \\
\hline \multicolumn{3}{|c|}{ dose per fraction (Gy RBE) } \\
\hline Mean & 1.8 & \\
\hline Median & 1.8 & \\
\hline Standard deviation & 0.2 & \\
\hline Quartile 1-quartile 3 & $1.8-1.8$ & \\
\hline Minimum - maximum & $1.8-2$ & \\
\hline \multicolumn{3}{|l|}{ Number of fractions } \\
\hline Mean & 29 & \\
\hline Median & 30 & \\
\hline Standard deviation & 3 & \\
\hline Quartile 1-quartile 3 & $30-31$ & \\
\hline Minimum-maximum & $15-32$ & \\
\hline \multicolumn{3}{|l|}{ Fractions per week } \\
\hline 5 fractions per week & 23 & [51.1\%] \\
\hline 6 fractions per week & 22 & [48.9\%] \\
\hline \multicolumn{3}{|c|}{ Proton beam radiotherapy technique } \\
\hline IMPT & 37 & [82.2\%] \\
\hline $\mathrm{SBO}$ & 8 & {$[17.8 \%]$} \\
\hline \multicolumn{3}{|l|}{ Number of beams } \\
\hline 1 & 2 & {$[4.4 \%]$} \\
\hline 2 & 27 & [55.6\%] \\
\hline 3 & 19 & [40.0\%] \\
\hline \multicolumn{3}{|l|}{ GTV (ml) } \\
\hline Mean & 8 & \\
\hline Median & 5 & \\
\hline Standard deviation & 10 & \\
\hline Quartile 1-quartile 3 & $3-8$ & \\
\hline Minimum-maximum & $0.3-60$ & \\
\hline \multicolumn{3}{|l|}{ PTV (ml) } \\
\hline Mean & 20 & \\
\hline Median & 12 & \\
\hline Standard deviation & 22 & \\
\hline Quartile 1-quartile 3 & $20-23$ & \\
\hline Minimum-maximum & $4-137$ & \\
\hline
\end{tabular}

Dmax, maximum dose; Dmean, mean dose; Gy RBE, gray relative biological effectiveness. 


\begin{tabular}{lcc} 
B) Doses for organs at risk (Gy RBE) & Dmax & Dmean \\
\hline Inner ear & & \\
Mean & 49.6 & 40.4 \\
Median & 54 & 43.8 \\
Quartile 1-quartile 3 & $51.1-55.4$ & $38-50.3$ \\
$\quad$ Minimum-maximum & $0-59.4$ & $0-57.3$ \\
Adjacent cranial nerves & & \\
Mean & 8.6 & 3.1 \\
Median & 2.4 & 0.4 \\
Quartile 1-quartile 3 & $0.9-9.3$ & $0.1-1.2$ \\
Minimum-maximum & $0.3-51.9$ & $0-45.5$ \\
Ipsilateral temporal lobe & & \\
Mean & 50.3 & 13.6 \\
Median & 53.2 & 13.6 \\
Quartile 1-quartile 3 & $50.3-54.8$ & $12.2-16.4$ \\
$\quad$ Minimum-maximum & $3.7-58.3$ & $0-19.8$ \\
Brain stem & & \\
Mean & 51.4 & 12 \\
Median & 53.5 & 10.6 \\
Quartile 1-quartile 3 & $52-54.6$ & $6.1-14.9$ \\
Minimum-maximum & $20.3-57.9$ & $0.5-44.9$ \\
Ventricular system & & \\
Mean & 55.1 & 6.1 \\
Median & 55.1 & 6.1 \\
Quartile 1-quartile 3 & $55.1-55.1$ & $6.1-6.1$ \\
Minimum-maximum & $55.1-55.1$ & $6.1-6.1$ \\
\hline
\end{tabular}

Dmax, maximum dose; Dmean, mean dose; Gy RBE, Gray Relative Biological Effectiveness.

TABLE 3 | Treatment effectiveness observation.

\begin{tabular}{lcc}
\hline \multicolumn{3}{c}{$\mathbf{n}=\mathbf{4 5}[\%]$} \\
\hline Time PRT end until first imaging follow-up (weeks) \\
Mean & 9 & \\
Median & 8 & \\
Standard deviation & 9 & \\
Quartile 1-quartile 3 & $6-10$ & \\
Minimum-maximum & $1-44$ & \\
Total follow-up period (months) & & \\
Mean & 43 & \\
Median & 42 & \\
Standard deviation & 25 & {$[0.0 \%]$} \\
Quartile 1-quartile 3 & $26-61$ & {$[6.7 \%]$} \\
Minimum-maximum & $3-97$ & {$[93.3 \%]$} \\
Response to radiotherapy during follow-up period & {$[0.0 \%]$} \\
Complete remission & 0 & \\
Partial remission & 3 & {$[100 \%]$} \\
Stable disease & 42 & {$[0.0 \%]$} \\
Progressive disease & 0 & \\
Progression-free survival & & \\
Yes & 45 & \\
No & 0 & \\
\hline
\end{tabular}

glossopharyngeal nerve as well as the vagal nerve, each with $4.4 \%$. A neurofibromatosis type 2 as a risk factor for schwannoma occurrence was found in $11.1 \%$ of the patients. Between primary diagnosis and radiotherapy start passed in a median of 18 months (range: 2 months to 12 years). Diagnosis was made mainly by imaging with MRI. In $37.8 \%$ of the cases, histology was confirmed by a prior biopsy or resection. In two cases (4.4\%), a DOTATOC-PET-CT was needed to exclude meningioma. In $57.8 \%$ of the patients, treatment was indicated due to observed progression. About $95.6 \%$ of all treated patients complained restrictions in everyday life due to schwannoma symptoms. In median, schwannoma size or gross target volume (GTV) was $5 \mathrm{ml}$ at radiotherapy start ranging from 0.3 to $60 \mathrm{ml}$. The planning target volume (PTV) was $12 \mathrm{ml}$ in median, ranging from 4 to $137 \mathrm{ml}$. The median total dose was $54 \mathrm{~Gy}$ (range: $40-$ $57.6 \mathrm{~Gy}$ ) with a median single dose of $1.8 \mathrm{~Gy}$ (range 1.8-2 Gy), applied in five to six fractions per week. Two patients were treated with reduced doses due to pre-irradiation in the past. The most commonly used technique was intensity modulated proton therapy (IMPT) with $82.2 \%$, followed by single beam optimization (SBO) with $17.8 \%$. Between one and three beams were used with two beams in $60.0 \%$, three beams in $42.2 \%$, and one beam in $4.4 \%$.

Detailed patient characteristics are presented in Table 1; detailed treatment characteristics are presented in Table 2.

\section{Efficacy and Toxicity}

The follow-up period was 42 months in median (IQR 26-61). In median after 2 months (range: $0.25-11$ months), the first follow-up MRI was performed. During the entire follow-up period, $93.3 \%$ of the patients showed stable disease, and $6.7 \%$ demonstrated partial remission. None of the patients had a schwannoma progression in the observation period. Detailed data on treatment effectiveness observation are presented in Table 3.

Before radiotherapy start and during the follow-up period, detailed data on clinical signs and symptoms caused by schwannoma were recorded via repeated medical history and physical exam assessment at each follow-up visit. This allows for a longitudinal presentation in the course of time of multiple specific symptoms as presented in Table 4 . At radiotherapy start, $95.6 \%$ reported clinical symptoms due to the schwannoma. With vestibular schwannoma being predominantly observed, most patients complained about hearing impairment (80.0\%), followed by vertigo (35.6\%) and tinnitus (22.4\%). A trigeminal neuralgia was reported by $15.6 \%$ of the patients, and $4.4 \%$ of the patients suffered from difficulty swallowing. All symptoms were graded analogous to CTCAE grading for better comparability with follow-up findings even if CTCAE grading was not developed to assess pretherapeutic symptoms. After PRT, $60 \%$ of the patients had stable symptoms, $4.4 \%$ of the patients reported a symptom improvement, and $35.6 \%$ of the patients reported any symptom deterioration after PRT, mainly transient fatigue. New or worsening cranial nerve dysfunctions were found in $20.0 \%$ of all the patients in the follow-up period, e.g., a worsening in tinnitus, but never relevant for activities of daily life. The reported symptoms described above were mild and graded CTCAE I-II. No CTCAE ${ }^{\circ} I I / I V$ toxicities were observed. Symptoms and toxicities that were not associated to cranial nerves like fatigue, headache, skin toxicity, dysgeusia, and alopecia were mainly observed in the early post-irradiation period and resolved during further follow-up. Explorative analysis via logistic regression modeling could not find any substantially influencing factors among all descriptively presented variables on the improvement or deterioration of clinical symptoms. 
TABLE 4 | Long-term treatment toxicity assessment.

\begin{tabular}{|c|c|c|c|c|c|c|c|c|c|c|c|c|c|c|c|c|}
\hline \multirow[t]{3}{*}{ Clinical symptom } & \multicolumn{4}{|c|}{ Pre-irradiation } & \multicolumn{4}{|c|}{ Early post-irradiation } & \multicolumn{4}{|c|}{ Late post-irradiation } & \multicolumn{4}{|c|}{ Overall post-irradiation } \\
\hline & \multicolumn{2}{|c|}{$\begin{array}{l}\text { Low grade* } \\
\text { (CTCAE I-II) }\end{array}$} & \multicolumn{2}{|c|}{$\begin{array}{l}\text { High grade* } \\
\text { (CTCAE } \geq I I I)\end{array}$} & \multicolumn{2}{|c|}{$\begin{array}{l}\text { Low grade } \\
\text { (CTCAE I-II) }\end{array}$} & \multicolumn{2}{|c|}{$\begin{array}{l}\text { High grade } \\
\text { (CTCAE } \geq I I I)\end{array}$} & \multicolumn{2}{|c|}{$\begin{array}{l}\text { Low grade } \\
\text { (CTCAE I-II) }\end{array}$} & \multicolumn{2}{|c|}{$\begin{array}{l}\text { High grade } \\
\text { (CTCAE } \geq I I I)\end{array}$} & \multicolumn{2}{|c|}{ Improvement } & \multicolumn{2}{|c|}{ deterioration } \\
\hline & $\mathbf{n}$ & {$[\%]$} & $\mathbf{n}$ & [\%] & $\mathbf{n}$ & {$[\%]$} & $\mathbf{n}$ & {$[\%]$} & $\mathbf{n}$ & {$[\%]$} & $\mathbf{n}$ & {$[\%]$} & $\mathbf{n}$ & {$[\%]$} & $\mathbf{n}$ & {$[\%]$} \\
\hline Any & 43 & [95.6\%] & 0 & [0.0\%] & 45 & [100\%] & 0 & [0.0\%] & 45 & [100\%] & 0 & [0.0\%] & 0 & [0.0\%] & 16 & [35.6\%] \\
\hline \multicolumn{17}{|l|}{ Cranial nerves } \\
\hline Olfactory nerve & 0 & [0.0\%] & 0 & [0.0\%] & 0 & [0.0\%] & 0 & [0.0\%] & 0 & [0.0\%] & 0 & [0.0\%] & 0 & [0.0\%] & 0 & [0.0\%] \\
\hline Optic nerve & 0 & [0.0\%] & 0 & [0.0\%] & 0 & [0.0\%] & 0 & [0.0\%] & 0 & [0.0\%] & 0 & [0.0\%] & 0 & [0.0\%] & 0 & {$[0.0 \%]$} \\
\hline Oculomotory nerve & 0 & [0.0\%] & 0 & [0.0\%] & 0 & [0.0\%] & 0 & [0.0\%] & 0 & [0.0\%] & 0 & [0.0\%] & 0 & [0.0\%] & 0 & [0.0\%] \\
\hline Trochlear nerve & 0 & [0.0\%] & 0 & [0.0\%] & 0 & [0.0\%] & 0 & [0.0\%] & 0 & [0.0\%] & 0 & [0.0\%] & 0 & [0.0\%] & 0 & [0.0\%] \\
\hline Trigeminal nerve & 7 & [15.6\%] & 0 & [0.0\%] & 9 & [20.0\%] & 0 & [0.0\%] & 9 & [20.0\%] & 0 & [0.0\%] & 0 & [0.0\%] & 3 & [6.7\%] \\
\hline Abducens nerve & 0 & [0.0\%] & 0 & [0.0\%] & 0 & [0.0\%] & 0 & [0.0\%] & 0 & [0.0\%] & 0 & [0.0\%] & 0 & [0.0\%] & 0 & [0.0\%] \\
\hline Facial nerve & 11 & [26.7\%] & 0 & {$[0.0 \%]$} & 12 & [26.7\%] & 0 & [0.0\%] & 12 & [26.7\%] & 0 & [0.0\%] & 0 & [0.0\%] & 1 & [2.2\%] \\
\hline Vestibulocochlear nerve & 37 & [82.2\%] & 0 & [0.0\%] & 37 & [82.2\%] & 0 & [0.0\%] & 37 & [82.2\%] & 0 & [0.0\%] & 2 & [4.4\%] & 5 & [11.1\%] \\
\hline Tinnitus & 11 & [22.4\%] & 0 & [0.0\%] & 12 & [26.7\%] & 0 & [0.0\%] & 12 & [26.7\%] & 0 & [0.0\%] & 1 & [2.2\%] & 5 & [11.1\%] \\
\hline Hearing impairment & 36 & {$[80.0 \%]$} & 0 & [0.0\%] & 36 & {$[80.0 \%]$} & 0 & {$[0.0 \%]$} & 36 & {$[80.0 \%]$} & 0 & {$[0.0 \%]$} & 0 & {$[0.0 \%]$} & 0 & {$[0.0 \%]$} \\
\hline Vertigo & 16 & [35.6\%] & 0 & [0.0\%] & 16 & [35.6\%] & 0 & [0.0\%] & 16 & [35.6\%] & 0 & [0.0\%] & 2 & [4.4\%] & 3 & {$[6.7 \%]$} \\
\hline Glossopharyngeal nerve & 1 & [2.2\%] & 0 & [0.0\%] & 1 & [2.2\%] & 0 & [0.0\%] & 1 & [2.2\%] & 0 & [0.0\%] & 0 & [0.0\%] & 0 & [0.0\%] \\
\hline Vagal nerve & 1 & [2.2\%] & 0 & [0.0\%] & 1 & [2.2\%] & 0 & {$[0.0 \%]$} & 1 & [2.2\%] & 0 & [0.0\%] & 0 & [0.0\%] & 0 & [0.0\%] \\
\hline Accessory nerve & 0 & [0.0\%] & 0 & [0.0\%] & 0 & [0.0\%] & 0 & [0.0\%] & 0 & [0.0\%] & 0 & [0.0\%] & 0 & [0.0\%] & 0 & [0.0\%] \\
\hline Hypoglossal nerve & 0 & [0.0\%] & 0 & [0.0\%] & 0 & [0.0\%] & 0 & [0.0\%] & 0 & [0.0\%] & 0 & [0.0\%] & 0 & [0.0\%] & 0 & [0.0\%] \\
\hline RICE & 0 & [0.0\%] & 0 & [0.0\%] & 0 & [0.0\%] & 0 & [0.0\%] & 7 & [15.6\%] & 0 & [0.0\%] & 0 & [0.0\%] & 7 & [15.6\%] \\
\hline \multicolumn{17}{|l|}{ Others } \\
\hline Fatigue & 0 & [0.0\%] & 0 & [0.0\%] & 11 & [24.4\%] & 0 & [0.0\%] & 3 & {$[6.7 \%]$} & 0 & [0.0\%] & 0 & [0.0\%] & 11 & [24.4\%] \\
\hline Headache & 0 & [0.0\%] & 0 & [0.0\%] & 5 & [11.1\%] & 0 & [0.0\%] & 1 & [2.2\%] & 0 & [0.0\%] & 0 & [0.0\%] & 5 & [11.1\%] \\
\hline Skin toxicity & 0 & [0.0\%] & 0 & [0.0\%] & 1 & [2.2\%] & 0 & [0.0\%] & 0 & [0.0\%] & 0 & [0.0\%] & 0 & [0.0\%] & 1 & [2.2\%] \\
\hline Dysgeusia & 0 & [0.0\%] & 0 & [0.0\%] & 1 & [2.2\%] & 0 & [0.0\%] & 0 & [0.0\%] & 0 & [0.0\%] & 0 & [0.0\%] & 1 & [2.2\%] \\
\hline Alopecia & 0 & [0.0\%] & 0 & [0.0\%] & 4 & [8.9\%] & 0 & [0.0\%] & 0 & [0.0\%] & 0 & [0.0\%] & 0 & [0.0\%] & 4 & [8.9\%] \\
\hline
\end{tabular}

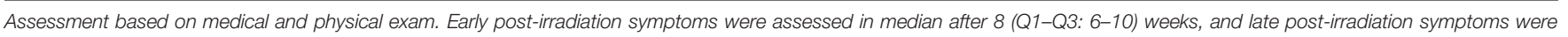

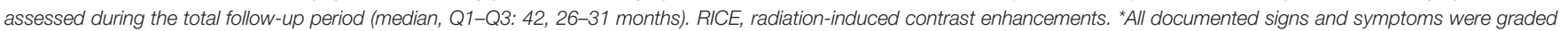
analogous to CTCAE grading for better comparability. CTCAE, common terminology criteria for adverse events.

No secondary malignancies were encountered during the follow-up period.

\section{RICE}

The follow-up MRIs revealed a contrast enhancement in brain parenchyma after PRT consistent with an RICE in seven cases (15.6\%). These lesions were observed after a median of 14 months (range: 2-26 months) after PRT. In two of the seven RICE cases (29\%), a deterioration of clinical symptoms was observed; in one case, mild gait disturbances and in the other case a facial paresthesia were reported, which made an outpatinet treatment with a short course of orally administered necessary (both CTCAE ${ }^{\circ} \mathrm{II}$ ). Due to sufficient response to corticosteroid treatment, in no case bevacizumab administration as an antiVEGF antibody was needed. Three of the seven lesions (43\%) regressed during the follow-up period, and no lesion showed progression. Further analysis of RICE cases did not reveal considerable differences in the subgroup, in which RICE occurred, compared to the subgroup, in which no RICE was diagnosed. Patients with RICE had been treated with standard therapy of 54-57.6 Gy in 1.8-2.0 Gy RBE per fraction with five to six fractions per week. GTV volume was 2-7 ml. Only one patient underwent previous surgery; none of the patients had neurofibromatosis type 2. CI for PTV was in the median of 0.99 (range $0.98-1.0$ ). Due to lack of power, explorative analysis via logistic regression modeling could not find any influencing factors on the development of RICE by comparing the 7 RICE cases to the 38 cases without RICE among the tested variables: age $>55$ years, tumor volume $\geq 5 \mathrm{ml}$, technique (IMPT vs. SBO), number of beams, and fractions per week. Table 5 presents a detailed workup of RICE. Table 5A demonstrates an overview analysis on all RICE cases, whereas Table 5B shows a detailed descriptive analysis of each specific RICE case. Table 5C shows the logistic regression model for RICE risk factors. Figure 1 shows representative images for all seven cases.

\section{DISCUSSION}

This study investigates the effectiveness and toxicity of fractionated proton beam radiotherapy for cranial nerve schwannomas.

As expected, most cranial nerve schwannomas affected the vestibulocochlear nerve, and therefore, most patients presenting to our clinic suffered from hearing impairment, tinnitus, or vertigo. Nevertheless, cranial nerve schwannomas originating from the trigeminal nerve, the glossopharyngeal nerve, and the vagal nerve were also found, mainly associated to neurofibromatosis type 2 as supported by literature data (24). Most patients sought for treatment due to clinical symptoms or a documented schwannoma progression under monitoring.

Proton beam radiotherapy for cranial nerve schwannoma was shown to be effective. No schwannoma progression was 
observed, and therefore, $100 \%$ effectivity can be reported for the follow-up period of 3.5 years in median. These effectivity rates for proton beam radiotherapy are very promising. However, further prolongation of the follow-up period is needed. The only data available on PRT in vestibular schwannoma include 95

TABLE 5 | Detailed analysis of RICE ( $\mathrm{n}=7$ [15.6\%]).

\begin{tabular}{|c|c|c|}
\hline A) Overall RICE analysis & \multicolumn{2}{|c|}{$n=7[16 \%]$} \\
\hline \multicolumn{3}{|c|}{ Time radiotherapy end to first occurrence of RICE (months) } \\
\hline Mean & 14 & \\
\hline Median & 14 & \\
\hline Quartile 1-quartile 3 & $12-16$ & \\
\hline Minimum-maximum & $2-26$ & \\
\hline \multicolumn{3}{|l|}{ Symptomatic RICE } \\
\hline Yes & 2 (CTCAE II) & [28.6\%] \\
\hline No & 5 & {$[71.4 \%]$} \\
\hline \multicolumn{3}{|l|}{ Treatment needed for RICE } \\
\hline Steroids only & 7 & [100\%] \\
\hline Bevacizumab (anti-VEGF antibody) & 0 & {$[0 \%]$} \\
\hline \multicolumn{3}{|c|}{ Observed regression during follow-up period } \\
\hline Yes & 3 & [42.9\%] \\
\hline No & 4 & [57.1\%] \\
\hline \multicolumn{3}{|c|}{ Observed progression during follow-up period } \\
\hline Yes & 0 & {$[0 \%]$} \\
\hline No & 7 & [100\%] \\
\hline
\end{tabular}

RICE, radiation-induced contrast enhancements. patients treated from March 1991 to March 2008 at Loma Linda University Medical Center. Fractionated proton radiotherapy at daily doses of $1.8 \mathrm{~Gy}$ and a total dose ranging between $59.6 \mathrm{~Gy}$ (RBE) and 50.4 Gy (RBE) was employed, depending on hearing function. Local control rates for the median follow-up time of 64 months were 95-92\%, depending on the applied dose. Cranial nerve injuries occurred in two patients. Hearing preservation was maintained in $44-64 \%$ of the patients, depending on the applied dose. The overall patient cohort was divided into three groups with graduated dose concepts (16).

For gamma knife, a control rate of $98 \%$ after a median followup period of 5.8 years was reported (25). For the "watch-andwait" strategy, a meta-analysis demonstrated in median $43 \%$ of schwannoma progressions in a similar follow-up period of 3.2 years (26). For surgery, a median of $9 \%$ schwannoma progression was demonstrated in a follow-up of 3.1 years (27) with a considerable rate of surgery-associated cranial nerve damage with chances of hearing preservation between $47 \%$ and $88 \%$ and even death due to surgery-associated complications (28). Therefore, as stated by the EANO Guideline on the Diagnosis and Treatment of Vestibular Schwannoma, radiotherapy is superior to both the "watch-and-wait" strategy regarding efficacy as well as surgery especially regarding toxicity in small tumors $(<3 \mathrm{~cm})(9)$. But both the "watch-and-wait" strategy and surgery are valuable for selected patients. Patients without any symptoms or progression may be suitable for the "watch-and-

\begin{tabular}{|c|c|c|c|c|c|c|c|}
\hline \multicolumn{8}{|c|}{ B) Analysis of specific RICE cases } \\
\hline Case number & 1 & 2 & 3 & 4 & 5 & 6 & 7 \\
\hline Gender & Female & Male & Female & Male & Female & Female & Female \\
\hline Age at radiotherapy (years) & 63.9 & 82.4 & 67.7 & 62.8 & 79.3 & 77.8 & 44.3 \\
\hline Latency (months) & 17 & 14.9 & 2.3 & 13.8 & 25.7 & 10.3 & 13.3 \\
\hline Treatment & Steroids & Steroids & Steroids & Steroids & Steroids & Steroids & Steroids \\
\hline Inpatient treatment needed & No & No & No & No & No & No & No \\
\hline Cranial nerve & $\begin{array}{c}\text { Vestibulocochlear } \\
\text { nerve }\end{array}$ & $\begin{array}{l}\text { Vestibulocochlear } \\
\text { nerve }\end{array}$ & $\begin{array}{c}\text { Vestibulocochlear } \\
\text { nerve }\end{array}$ & $\begin{array}{c}\text { Vestibulocochlear } \\
\text { nerve }\end{array}$ & $\begin{array}{c}\text { Vestibulocochlear } \\
\text { nerve }\end{array}$ & $\begin{array}{c}\text { Vestibulocochlear } \\
\text { nerve }\end{array}$ & $\begin{array}{c}\text { Vestibulocochlear } \\
\text { nerve }\end{array}$ \\
\hline Previous surgery & No & No & No & No & No & No & Yes \\
\hline Neurofibromatosis type II & No & No & No & No & No & No & No \\
\hline Any pre-irradiation symptoms & Yes & Yes & Yes & Yes & Yes & Yes & Yes \\
\hline Pre-irradiation tinnitus & No & No & No & No & No & Yes & Yes \\
\hline $\begin{array}{l}\text { Pre-irradiation hearing } \\
\text { impairment }\end{array}$ & Yes & Yes & Yes & Yes & Yes & Yes & Yes \\
\hline Pre-irradiation vertigo & No & Yes & No & No & No & Yes & Yes \\
\hline $\begin{array}{l}\text { Any post-irradiation } \\
\text { deterioration }\end{array}$ & No & Yes & No & No & No & Yes & No \\
\hline Total dose (Gy RBE) & 54.0 & 54.0 & 54.0 & 57.6 & 57.6 & 54.0 & 57.6 \\
\hline Dose per fraction (Gy RBE) & 1.8 & 1.8 & 2.0 & 1.8 & 1.8 & 1.8 & 1.8 \\
\hline Fractions per week & 5 & 5 & 6 & 5 & 6 & 6 & 5 \\
\hline Radiotherapy technique & IMPT & $\mathrm{SBO}$ & IMPT & IMPT & IMPT & IMPT & IMPT \\
\hline Schwannoma size/GTV (ml) & 5 & 5 & 7 & 4 & 5 & 4 & 2 \\
\hline $\begin{array}{l}\text { Schwannoma maximal } \\
\text { diameter }(\mathrm{mm})\end{array}$ & 22 & 25 & 26 & 22 & 21 & 24 & 26 \\
\hline PTV (ml) & 10 & 12 & 20 & 9 & 12 & 9 & 5 \\
\hline $\begin{array}{l}\text { Maximum dose GTV (Gy } \\
\text { RBE) }\end{array}$ & 55.5 & 55.5 & 56.1 & 60.8 & 56.4 & 55.9 & 59.3 \\
\hline $\begin{array}{l}\text { Maximum dose brain stem } \\
\text { (Gy RBE) }\end{array}$ & 53.2 & 53.3 & 54.3 & 54.7 & 52.8 & 52.9 & 55.5 \\
\hline Number of beams & 3 & 2 & 2 & 3 & 3 & 3 & 3 \\
\hline Any pre-irradiation in the past & No & No & No & No & No & No & No \\
\hline
\end{tabular}

RICE, radiation-induced contrast enhancements. 


\begin{tabular}{lcc}
$\begin{array}{l}\text { C) Logistic regression analysis for } \\
\text { Variable }\end{array}$ & EsCE risk factors & \\
\hline Age $>55$ years & $-2.41(5.77--0.42)$ & p-value \\
Tumor volume $\geq 5 \mathrm{ml}$ & $-1.84(1.24-1.48)$ & 0.68 \\
Technique (IMPT vs. SBO) & $-0.61(1.50--0.40)$ & 0.14 \\
Number of beams & $1.77(1.17-1.51)$ & 0.69 \\
Fractions per week & $-0.72(1.01--0.72)$ & 0.13 \\
\end{tabular}

Cl, confidence interval IMPT, intensity-modulated proton therapy; SBO, single beam optimization; RICE, radiation-induced contrast enhancements.

wait" strategy, while very large tumors that have already completely destroyed the nerve and compress the brainstem may be suitable for surgery. For radiotherapy, there are several factors, e.g., dose, fractionation regimes, and treatment techniques, that have to be taken into consideration when deciding about the individually best approach. According to a retrospective comparative analysis of 125 patients in total, hearing preservation rates might be 2.5 -fold higher in fractionated radiotherapy than in single fraction stereotactic radiosurgery, but tumor control rates were at least $97 \%$ in both groups (29). However, literature demonstrates also data that report similar rates for hearing preservation and local control for both fractionated radiotherapy and single fraction stereotactic radiosurgery, and the dose of single fraction stereotactic radiosurgery was significantly influencing hearing preservation (30). Furthermore, according to literature, it is recommended to use single fraction stereotactic radiosurgery for smaller lesions, while FSRT can be used independently of tumor size and should be preferred in larger tumors (with contact to the brainstem) due to the better adherence to dose constraints with FSRT (30-33). For PRT, fractionated radiotherapy might be less toxic than single fraction stereotactic radiosurgery $(16,18)$. We cannot directly compare our results to stereotactic radiosurgery as our cohort was defined by unsuitability for stereotactic radiosurgery (T3-T4 tumors). Valid treatment alternatives for our cohort were surgery or fractionated photon radiotherapy. Cranial nerve preservation of the affected cranial nerve in fractionated proton/photon radiotherapy is well known to be superior to surgery. Compared to fractionated photon radiotherapy, fractionated proton radiotherapy spares the adjacent cranial nerves due to steep dose gradients given in proton radiotherapy. This is a dosimetric advantage, and literature is lacking data whether this results in clinically detectable advantages. Since cranial nerve neuromas are mainly located at the skull base often in close proximity to the brain stem or the facial nerve (for acoustic neuroma/vestibular schwannoma), sparing of adjacent structures is critical.

Interestingly, a retrospective analysis found lower tumor control rates in patients with neurofibromatosis type 2 compared to sporadic tumors (29). This was not confirmed by our study using fractionated PRT.

When patients present for radiotherapy, they are usually suffering from schwannoma-associated symptoms that are mainly hearing impairments in vestibular schwannoma. None of our patients reported a new or worsening hearing impairment after PRT despite relevant dose deposition on the inner ear
$\left(D_{\text {mean }}\right.$ in median $=43.8$ Gy $)$. Nevertheless, data on hearing function are based on medical history and physical exam so a very mild hearing deterioration might be underestimated in this analysis, since audiometry was not performed on a regular basis but only if symptoms were reported. Nevertheless, the used method is appropriate to detect a hearing deterioration that is in any way relevant to the patients' daily life. One-fifth of the patients reported any new or worsening mild clinical symptoms associated to the affected cranial nerve in the follow-up period with a worsening tinnitus being the most common observation. Symptoms like tinnitus or vertigo might represent an irritation of the irradiated cranial nerve. Even if some patients (4.4\%) reported an improvement of clinical symptoms, this analysis demonstrates that PRT can irritate or slightly impair the function of the affected cranial nerve leading to a mild deterioration of symptoms even if no loss of cranial nerve function was observed.

To sum up the scarce available data, fractionated proton beam radiotherapy for tumors unsuitable for stereotactic radiosurgery is a promising approach that should be further investigated. For detailed assessment of hearing function using repetitive audiometry, a prospective clinical trial is urgently needed. Such a trial is currently conducted at Boston using fractionated proton radiation therapy for vestibular schwannomas and will close recruitment in a couple of months, so results will be pending for multiple years (ClinicalTrials.gov Identifier: NCT01199978).

A special feature of this study is that RICE has been defined as an endpoint, and it was specifically evaluated for its occurrence, clinical presentation, treatment, and time course. Despite all advantageous properties of proton beam radiotherapy presented above, the rate of $16 \%$ for RICE needs to be further focused on. Due to close proximity of the irradiated cranial nerve schwannomas to the brain stem, RICE occurred in the vulnerable healthy brain stem tissue (Figure 2). This unfavorable localization was also the reason why RICE treatment was administered liberally, so all of our RICE cases received a short course of corticosteroids. In two (of seven) patients, RICE were mild symptomatic, but corticosteroids relieved symptoms quickly, so none of the patients needed a bevacizumab therapy. Anti-VEGF antibodies like bevacizumab go along with excellent remission rates for RICE and are therefore the treatment of choice in more severe cases of RICE $(34,35)$. We showed that these lesions occurred after a median of 14 months. In 43\%, the lesions regressed during the follow-up period. Due to this observed time course, we recommend a close timeline of follow-up including MRI within the first 2 years. For these $43 \%$ of cases, RICE is probably representing a radiationinduced blood-brain barrier disruption and probably not an irreversible radiation necrosis due to its transient nature, as supported by literature (36).

While the applied dose is standard (16), nearly half of our patients received up to six fractions per week, which is due to historical reasons and can therefore be regarded as slight acceleration. We decided at our institution not to proceed the irradiation with six fractions per week for central nervous system tumors as a safety measure. Nevertheless, this was not an influencing factor in the performed exploratory analysis 


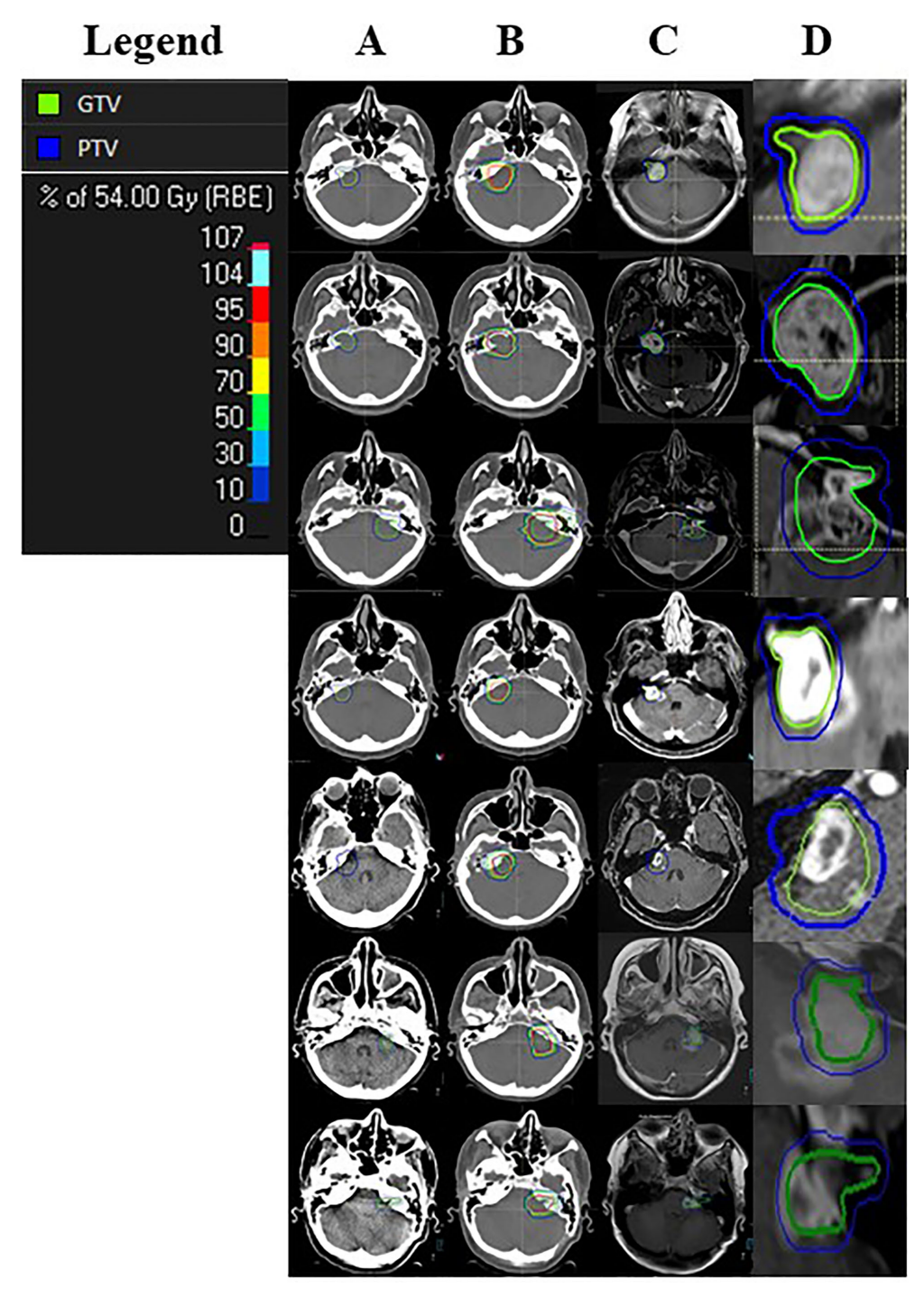

FIGURE 2 | Cases of Radiation-induced Contrast Enhancements (RICE). The image presents all seven cases of RICE observed in our study cohort. Column A presents the planning computer tomography with gross target volume (GTV, green) and planning target volume (PTV, blue) for each patient. Column B demonstrates the treatment plan with isodoses for each patient. Column C presents the follow-up magnetic resonance imaging (MRI) with the observed RICE at the time of its first notice and a projection of the initial GTV and PTV in this MRI with an enlargement of this region (column D).

regarding toxicity, RICE, or response of the treatment, but power was lacking. PTV conformity was sufficient in these cases, so inconformity can be excluded as a potential cause for RICE.

Another hypothesis is that the current technique of dose calculation for proton beam radiotherapy underestimates the real regional biological effectiveness as currently heavily discussed in the proton beam radiotherapy community. Interestingly, an RICE localization exactly behind the peak and therefore exactly distally to the target volume is a typical finding (as also depicted in Figure 1). The current method of dose prescription in PRT has known weaknesses. This leads to an uncertainty in dose calculation and therefore to underdosed and overdosed areas. Possibly, also an intrinsic difference in radiosensitivity of different brain tissue types might play a role and makes adaptions in proton beam radiotherapy planning necessary. First approaches for a physical risk model to estimate the probability of the occurrence of RICE for low-grade gliomas can be found in the literature (15). To reduce the risk for RICE without compromising the excellent oncologic outcomes, PRT planning needs to be improved further.

In summary, PRT was demonstrated to be effective and yielded high rates of cranial nerve functional preservation. The comparably low cranial nerve toxicity rates are promising, but the follow-up period needs to be further expanded. Also, a longer follow-up is needed. The analysis of RICE in this context is described for the first time. Even if no clinical relevance of RICE occurrence was demonstrated and corticosteroid response was good, it is above our understanding why some patients develop 
RICE and therefore further research is needed. Patients should undergo MRIs on a regular basis after cranial PRT.

\section{CONCLUSION}

These data demonstrate excellent effectiveness with 100\% local control in a median follow-up period of 3.6 years with a promising cranial nerve functional protection rate of $80 \%$. RICE occurred in $16 \%$ of the patients after PRT and were not or only mildly symptomatic but made a short course of dexamethasone necessary.

\section{DATA AVAILABILITY STATEMENT}

The raw data supporting the conclusions of this article will be made available by the authors, without undue reservation.

\section{ETHICS STATEMENT}

The studies involving human participants were reviewed and approved by Heidelberg University ethics committee, January

\section{REFERENCES}

1. Klekamp J, Samii M. Surgery of Spinal Nerve Sheath Tumors With Special Reference to Neurofibromatosis. Neurosurgery (1998) 42(2):279-89discussion 89-90. doi: 10.1097/00006123-199802000-00042

2. Fisher JL, Pettersson D, Palmisano S, Schwartzbaum JA, Edwards CG, Mathiesen T, et al. Loud Noise Exposure and Acoustic Neuroma. Am J Epidemiol (2014) 180(1):58-67. doi: 10.1093/aje/kwu081

3. Parry DM, Eldridge R, Kaiser-Kupfer MI, Bouzas EA, Pikus A, Patronas N. Neurofibromatosis 2 (NF2): Clinical Characteristics of 63 Affected Individuals and Clinical Evidence for Heterogeneity. Am J Med Genet (1994) 52(4):45061. doi: 10.1002/ajmg.1320520411

4. Mautner VF, Lindenau M, Baser ME, Hazim W, Tatagiba M, Haase W, et al. The Neuroimaging and Clinical Spectrum of Neurofibromatosis 2. Neurosurgery (1996) 38(5):880-5discussion 5-6. doi: 10.1097/00006123199605000-00004

5. Maertens O, Brems H, Vandesompele J, De Raedt T, Heyns I, Rosenbaum T, et al. Comprehensive NF1 Screening on Cultured Schwann Cells From Neurofibromas. Hum Mutat (2006) 27(10):1030-40. doi: 10.1002/humu.20389

6. Brosseau JP, Pichard DC, Legius EH, Wolkenstein P, Lavker RM, Blakeley JO, et al. The Biology of Cutaneous Neurofibromas: Consensus Recommendations for Setting Research Priorities. Neurology (2018) 91(2 Suppl 1):S14-s20. doi: 10.1212/WNL.0000000000005788

7. Gormley WB, Sekhar LN, Wright DC, Kamerer D, Schessel D. Acoustic Neuromas: Results of Current Surgical Management. Neurosurgery (1997) 41 (1):50-8; discussion 8-60. doi: 10.1097/00006123-199707000-00012

8. Samii M, Matthies C. Management of 1000 Vestibular Schwannomas (Acoustic Neuromas): Hearing Function in 1000 Tumor Resections. Neurosurgery (1997) 40(2):248-60; discussion 60-2. doi: 10.1097/00006123199702000-00005

9. Goldbrunner R, Weller M, Regis J, Lund-Johansen M, Stavrinou P, Reuss D, et al. EANO Guideline on the Diagnosis and Treatment of Vestibular Schwannoma. Neuro-Oncology (2020) 22(1):31-45. doi: 10.1093/neuonc/noz153

10. Lesueur P, Calugaru V, Nauraye C, Stefan D, Cao K, Emery E, et al. Proton Therapy for Treatment of Intracranial Benign Tumors in Adults: A Systematic Review. Cancer Treat Rev (2019) 72:56-64. doi: 10.1016/j.ctrv.2018.11.004
2018 (\#S-832/2018). Written informed consent for participation was not required for this study in accordance with the national legislation and the institutional requirements.

\section{AUTHOR CONTRIBUTIONS}

TE, LK, KH, and JD planned and supervised this analysis. TE performed data extraction and review. TE, RES, and DK performed all statistical analysis. TE reviewed data analysis and drafted the manuscript. All authors contributed patient data and participated in reviewing and improving analysis and manuscript. All authors read and approved the final manuscript.

\section{FUNDING}

We acknowledge financial support by Deutsche Forschungsgemeinschaft within the funding program for Open Access Publishing, by the Baden-Württemberg Ministry of Science, Research and the Arts and by Ruprecht-Karls-Universität Heidelberg. TE received funding by Ruprecht-Karls Universität Heidelberg, Herbert Kienzle Foundation, and Else Kröner-Fresenius Foundation.

11. Hall EJ. Intensity-Modulated Radiation Therapy, Protons, and the Risk of Second Cancers. Int J Radiat Oncol Biol Phys (2006) 65(1):1-7. doi: 10.1016/ j.ijrobp.2006.01.027

12. Sakthivel V, Ganesh KM, McKenzie C, Boopathy R, Selvaraj J. Second Malignant Neoplasm Risk After Craniospinal Irradiation in X-Ray-Based Techniques Compared to Proton Therapy. Australas Phys Eng Sci Med (2019) 42(1):201-9. doi: 10.1007/s13246-019-00731-y

13. König L, Haering P, Lang C, Splinter M, von Nettelbladt B, Weykamp F, et al. Secondary Malignancy Risk Following Proton vs. X-Ray Treatment of Mediastinal Malignant Lymphoma: A Comparative Modeling Study of Thoracic Organ-Specific Cancer Risk. Front Oncol (2020) 10:989. doi: 10.3389/fonc. 2020.00989

14. Bronk JK, Guha-Thakurta N, Allen PK, Mahajan A, Grosshans DR, McGovern SL. Analysis of Pseudoprogression After Proton or Photon Therapy of 99 Patients With Low Grade and Anaplastic Glioma. Clin Transl Radiat Oncol (2018) 9:30-4. doi: 10.1016/j.ctro.2018.01.002

15. Bahn E, Bauer J, Harrabi S, Herfarth K, Debus J, Alber M. Late Contrast Enhancing Brain Lesions in Proton-Treated Patients With Low-Grade Glioma: Clinical Evidence for Increased Periventricular Sensitivity and Variable RBE. Int J Radiat Oncol Biol Phys (2020) 107(3):571-8. doi: 10.1016/j.ijrobp.2020.03.013

16. Barnes CJ, Bush DA, Grove RI, Loredo LN, Slater JD. Fractionated Proton Beam Therapy for Acoustic Neuromas: Tumor Control and Hearing Preservation. Int J Particle Ther (2018) 4(4):28-36. doi: 10.14338/IJPT-1400014.1

17. Zhu S, Rotondo R, Mendenhall WM, Dagan R, Lewis D, Huh S, et al. LongTerm Outcomes of Fractionated Stereotactic Proton Therapy for Vestibular Schwannoma: A Case Series. Int J Particle Ther (2018) 4(4):37-46. doi: 10.14338/IJPT-17-00032.1

18. Weber DC, Chan AW, Bussiere MR, GRt H, Ancukiewicz M, Barker FG2nd, et al. Proton Beam Radiosurgery for Vestibular Schwannoma: Tumor Control and Cranial Nerve Toxicity. Neurosurgery (2003) 53(3):577-86; discussion 868. doi: 10.1227/01.NEU.0000079369.59219.C0

19. Emami B, Lyman J, Brown A, Coia L, Goitein M, Munzenrider JE, et al. Tolerance of Normal Tissue to Therapeutic Irradiation. Int J Radiat oncol biol Phys (1991) 21(1):109-22. doi: 10.1016/0360-3016(91)90171-Y 
20. Marks LB, Yorke ED, Jackson A, Ten Haken RK, Constine LS, Eisbruch A, et al. Use of Normal Tissue Complication Probability Models in the Clinic. Int J Radiat Oncol Biol Phys (2010) 76(3 Suppl):S10-9. doi: 10.1016/j.ijrobp.2009.07.1754

21. Shaw E, Kline R, Gillin M, Souhami L, Hirschfeld A, Dinapoli R, et al. Radiation Therapy Oncology Group: Radiosurgery Quality Assurance Guidelines. Int J Radiat Oncol Biol Phys (1993) 27(5):1231-9. doi: 10.1016/ 0360-3016(93)90548-A

22. Schwartz LH, Litière S, de Vries E, Ford R, Gwyther S, Mandrekar S, et al. RECIST 1.1-Update and Clarification: From the RECIST Committee. Eur J Cancer (Oxford England: 1990) (2016) 62:132-7. doi: 10.1016/j.ejca.2016.03.081

23. Schwartz LH, Seymour L, Litière S, Ford R, Gwyther S, Mandrekar S, et al. RECIST 1.1 - Standardisation and Disease-Specific Adaptations: Perspectives From the RECIST Working Group. Eur J Cancer (Oxford England: 1990) (2016) 62:138-45. doi: 10.1016/j.ejca.2016.03.082

24. Fisher LM, Doherty JK, Lev MH, Slattery WH3rd. Distribution of Nonvestibular Cranial Nerve Schwannomas in Neurofibromatosis 2. Otol neurotol: Off Publ Am Otol Society Am Neurotol Soc [and] Eur Acad Otol Neurotol (2007) 28(8):1083-90. doi: 10.1097/MAO.0b013e31815a8411

25. Tucker DW, Gogia AS, Donoho DA, Yim B, Yu C, Fredrickson VL, et al. Long-Term Tumor Control Rates Following Gamma Knife Radiosurgery for Acoustic Neuroma. World Neurosurgery (2019) 122:366-71. doi: 10.1016/ j.wneu.2018.11.009

26. Smouha EE, Yoo M, Mohr K, Davis RP. Conservative Management of Acoustic Neuroma: A Meta-Analysis and Proposed Treatment Algorithm. Laryngoscope (2005) 115(3):450-4. doi: 10.1097/00005537-200503000-00011

27. Sughrue ME, Kaur R, Rutkowski MJ, Kane AJ, Kaur G, Yang I, et al. Extent of Resection and the Long-Term Durability of Vestibular Schwannoma Surgery. J Neurosurg (2011) 114(5):1218-23. doi: 10.3171/2010.11.JNS10257

28. Samii M, Matthies C. Management of 1000 Vestibular Schwannomas (Acoustic Neuromas): The Facial Nerve-Preservation and Restitution of Function. Neurosurgery (1997) 40(4):684-94; discussion 94-5. doi: 10.1097/ 00006123-199704000-00006

29. Andrews DW, Suarez O, Goldman HW, Downes MB, Bednarz G, Corn BW, et al. Stereotactic Radiosurgery and Fractionated Stereotactic Radiotherapy for the Treatment of Acoustic Schwannomas: Comparative Observations of 125 Patients Treated at One Institution. Int J Radiat Oncol Biol Phys (2001) 50 (5):1265-78. doi: 10.1016/S0360-3016(01)01559-0

30. Combs SE, Welzel T, Schulz-Ertner D, Huber PE, Debus J. Differences in Clinical Results After LINAC-Based Single-Dose Radiosurgery Versus Fractionated Stereotactic Radiotherapy for Patients With Vestibular Schwannomas. Int J Radiat Oncol Biol Phys (2010) 76(1):193-200. doi: 10.1016/j.ijrobp.2009.01.064

31. Combs SE, Engelhard C, Kopp C, Wiedenmann N, Schramm O, Prokic V, et al. Long-Term Outcome After Highly Advanced Single-Dose or Fractionated Radiotherapy in Patients With Vestibular Schwannomas Pooled Results From 3 Large German Centers. Radiotherapy Oncol (2015) 114(3):378-83. doi: 10.1016/j.radonc.2015.01.011

32. Kessel KA, Fischer H, Vogel MM, Oechsner M, Bier H, Meyer B, et al. Fractionated vs. Single-Fraction Stereotactic Radiotherapy in Patients With Vestibular Schwannoma: Hearing Preservation and Patients' Self-Reported Outcome Based on an Established Questionnaire. Strahlentherapie und Onkologie: Organ der Deutschen Rontgengesellschaft [et al] (2017) 193 (3):192-9. doi: 10.1007/s00066-016-1070-0
33. Wagner J, Welzel T, Habermehl D, Debus J, Combs SE. Radiotherapy in Patients With Vestibular Schwannoma and Neurofibromatosis Type 2: Clinical Results and Review of the Literature. Tumori (2014) 100(2):189-94. doi: 10.1177/030089161410000212

34. Gonzalez J, Kumar AJ, Conrad CA, Levin VA. Effect of Bevacizumab on Radiation Necrosis of the Brain. Int J Radiat Oncol Biol Phys (2007) 67 (2):323-6. doi: 10.1016/j.ijrobp.2006.10.010

35. Zhuang H, Shi S, Yuan Z, Chang JY. Bevacizumab Treatment for Radiation Brain Necrosis: Mechanism, Efficacy and Issues. Mol Cancer (2019) 18(1):21. doi: 10.1186/s12943-019-0950-1

36. Rubin P, Gash DM, Hansen JT, Nelson DF, Williams JP. Disruption of the Blood-Brain Barrier as the Primary Effect of CNS Irradiation. Radiotherapy Oncol (1994) 31(1):51-60. doi: 10.1016/0167-8140(94)90413-8

Conflict of Interest: TE reports grants from Ruprecht-Karls Universität Heidelberg, Herbert Kienzle Foundation, and Else Kröner-Fresenius Foundation and received travel reimbursement from Bristol-Myers Squibb outside the submitted work. JH-R received speaker fees and travel reimbursement from ViewRay Inc, as well as travel reimbursement and grants from IntraOP Medical and Elekta Instrument AB outside the submitted work. RS reports grants from Ruprecht-Karls Universität Heidelberg, during the conduct of the study; personal fees from Accuray Inc., personal fees from AstraZeneca GmbH, personal fees from Bristol Myers Squibb GmbH \& Co., personal fees from Novocure GmbH, personal fees from Merck KGaA, personal fees from Takeda GmbH, and grants from Accuray Inc., outside the submitted work. JD reports grants from the Clinical Research Institute (CRI), grants from View Ray Incl., grants from Accuray International, grants from Accuray Incorporated, grants from RaySearch Laboratories AB, grants from Vision RT limited, grants from Merck Serono GmbH, grants from Astellas Pharma GmbH, grants from Astra Zeneca $\mathrm{GmbH}$, grants from Siemens Healthcare $\mathrm{GmbH}$, grants from Solution Akademie GmbH, grants from Eromed PLC Surrey Research Park, grants from Quintiles GmbH, grants from Pharmaceutical Research Associates GmbH, grants from Boehringer Ingelheim Pharma $\mathrm{GmbH} \mathrm{Co}$, grants from PTW-Frieburg Dr. Pychlau GmbH, grants from Nanobiotix A.a., grants from IntraOP Medical, outside the submitted work. LK reports grants from Ruprecht-Karls Universität Heidelberg, personal fees from Accuray Inc., and Novocure GmbH outside the submitted work.

The remaining authors declare that the research was conducted in the absence of any commercial or financial relationships that could be construed as a potential conflict of interest.

Publisher's Note: All claims expressed in this article are solely those of the authors and do not necessarily represent those of their affiliated organizations, or those of the publisher, the editors and the reviewers. Any product that may be evaluated in this article, or claim that may be made by its manufacturer, is not guaranteed or endorsed by the publisher.

Copyright (๑) 2021 Eichkorn, Regnery, Held, Kronsteiner, Hörner-Rieber, El Shafie, Herfarth, Debus and König. This is an open-access article distributed under the terms of the Creative Commons Attribution License (CC BY). The use, distribution or reproduction in other forums is permitted, provided the original author(s) and the copyright owner(s) are credited and that the original publication in this journal is cited, in accordance with accepted academic practice. No use, distribution or reproduction is permitted which does not comply with these terms. 